\title{
Fibronectin Conformation and Assembly: Analysis of Fibronectin Deletion Mutants and Fibronectin Glomerulopathy (GFND) Mutants
}

\author{
Tomoo Ohashi, ${ }^{*}$ C Christopher A. Lemmon, ${ }^{\dagger}$ and Harold P. Erickson* \\ Department of Cell Biology, Duke University Medical Center, Durham, North Carolina 27710, United States
}

\begin{abstract}
To study fibronectin (FN) conformation and assembly, we generated several deletion mutants: $\mathrm{FN} \Delta^{\mathrm{I}} 1-5, \mathrm{FN} \Delta^{\mathrm{III}} 1-3, \mathrm{FN} \Delta^{\mathrm{III}} 4-8$, and FN $\Delta^{\mathrm{III}} 11-14$. A monomeric form, FNmono, which lacked the C-terminal dimerization region, was also created. FNtnA-D was generated by swapping FNIII domains $1-8$ in FN $\Delta^{\mathrm{III}} 11-14$ with seven FNIII domains from tenascin-C. The conformations of these mutants were analyzed by glycerol gradient sedimentation under low-salt $(20 \mathrm{mM} \mathrm{NaCl})$ and high-salt $(200 \mathrm{mM} \mathrm{NaCl})$ conditions. Surprisingly, most of the mutants showed a compact conformation under low-salt conditions, except for FNtnA-D. When we tested these mutants in cell culture, FN $\Delta^{\mathrm{I}} 1-5, \mathrm{FN} \Delta^{\mathrm{III}} 1-3$, and FNtnA-D were unable to form a matrix. Interestingly, $\mathrm{FN} \Delta^{\mathrm{III}} 1-3$ and FNtnA-D were capable of co-assembly with full-length FN, while FN $\Delta^{\mathrm{I}} 1-$ 5 was not. This indicates that the segment ${ }^{\mathrm{I}} 1-5$ is crucial for matrix assembly and segment ${ }^{\mathrm{III}} 1-3$ is also important. Mutations in FN are associated with glomerulopathy, but when we studied mutant proteins, the single-nucleotide mutations had only minor effects on conformation and matrix assembly. The mutations may destabilize their FNIII domains or generate dimers of dimers by disulfide cross-linking.
\end{abstract}

$\mathrm{P}$ lasma fibronectin (FN) is secreted by hepatocytes in the liver and circulates in blood. ${ }^{1}$ Plasma FN is a dimer, each subunit comprising $12 \mathrm{FN}$ type I domains, two FN type II domains, 15 FN type III domains, and a variable domain (V), although one of the subunits in the dimer lacks $\mathrm{V}$ because of alternative splicing. ${ }^{2}$ The conformation of plasma $\mathrm{FN}$ has been extensively studied over the past several decades. It forms a compact conformation under physiological conditions, but at high ionic strength or high $\mathrm{pH}$, the conformation changes to an extended form. ${ }^{3-6}$ The exact structure of this compact conformation is not known and may represent multiple states; in this paper, we use the term "compact conformation" to refer to the state (or states) of the protein that sediments faster at a low ionic strength. It has been proposed that an interaction between ${ }^{\mathrm{III}} 2-3$ and ${ }^{\mathrm{III}} 12-14$ induces the compact conformation. ${ }^{7}$ This interaction was originally discovered by the dimerization of FN fragment ${ }^{\text {III }} 2-14$. It is important to note that this dimerization does not occur at a physiological salt concentration $(150 \mathrm{mM}$ $\mathrm{NaCl}$ ), which suggests that the compact conformation is stabilized by interactions in addition to ${ }^{\mathrm{III}} 2-3$ and ${ }^{\mathrm{III}} 12-14$. An earlier study indicated that ${ }^{\mathrm{I}} 1-5$ interacted with ${ }^{\mathrm{III}} 12-14$ to form the compact conformation. ${ }^{8}$ An interaction between ${ }^{\mathrm{I}} 4-5$ and ${ }^{\text {III }} 3$ was also suggested, ${ }^{9}$ although this interaction was extremely weak at a physiological salt concentration. Maurer et al. ${ }^{10}$ recently showed that a ${ }^{\mathrm{I}} 1-5$ binding peptide, which was engineered from a bacterial adhesin, induced the extended FN conformation in solution. This also suggests that ${ }^{\mathrm{I}} 1-5$ is involved in forming the compact conformation.

To study the function of plasma FN, tissue specific knockout mice were created to deplete FN in blood. ${ }^{11}$ Surprisingly, these mice did not show any obvious abnormality and had normal skin wound healing. However, transient focal cerebral ischemia was more severe in the knockout mice, indicating that plasma FN plays a role in brain wound healing. Plasma FN is also important for thrombus initiation, growth, and stability in injured arterioles. $^{12}$

Soluble FN assembles into insoluble fibrils, called an FN matrix, on the cell surface. Detergent-insoluble FN matrix fibrils appear to be formed by noncovalent bonds. ${ }^{13,14}$ However, the exact locations of potential bonds and the interfaces between molecules are still not known. Because soluble FN, such as plasma FN, cannot assemble without cells, it is thought that one or more cryptic assembly sites are exposed only when FN binds to the cell surface. Electrostatic bonds between dimers might account for noncovalently bonded multimers, although these bonds may not be strong enough to hold the molecules together during detergent extraction. We have previously proposed that FN aggregation and assembly require the unfolding of FNIII domains. ${ }^{15,16}$ In this model, high local FN concentrations on the cell surface could enhance the probability of interactions following the spontaneous unfolding and/or opening of FNIII domains.

A recent genetic study showed that a hereditary kidney disease, FN glomerulopathy [or glomerulopathy with FN deposits (GFND)], was caused by mutations in the FN1 gene. ${ }^{17}$ Singlenucleotide polymorphisms were found in domains ${ }^{\mathrm{III}} 4$ and ${ }^{\mathrm{III}} 13$.

Received: June 21, 2017

Revised: July 24, 2017

Published: July 26, 2017 


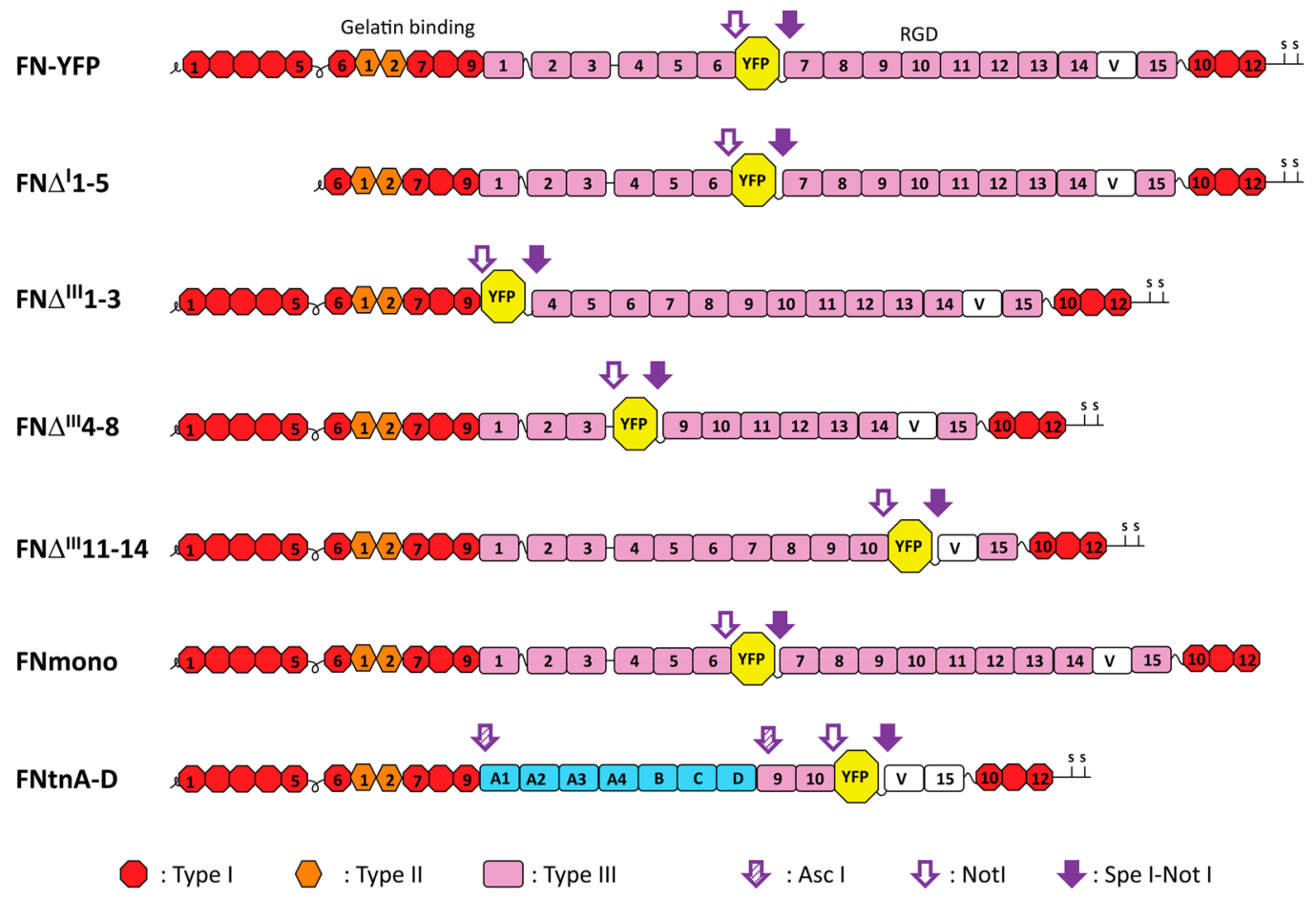

Figure 1. Diagrams of FN-YFP and the deletion mutants used in this study. Predicted unstructured regions are shown as lines. The locations of engineered restriction enzyme sites for cloning are shown with arrows.

Because nonfibrillar FN deposits in GFND patients were mainly derived from plasma $\mathrm{FN}$, the authors suggested that these mutations may affect the conformation of plasma $\mathrm{FN}$, which more easily formed abnormal nonfibrillar FN deposits in the glomerular matrix. More recently, additional mutations were found in ${ }^{\mathrm{II}} 4$ and ${ }^{\mathrm{III}} 13$, and one mutation was found in ${ }^{\mathrm{III}} 9$. $^{18}$

In the study presented here, we generated several $\mathrm{FN}$ deletion mutants and GFND mutants, to study the domains responsible for forming the compact FN conformation and assembling the matrix.

\section{MATERIALS AND METHODS}

Mutant Construction and Mammalian Cell Protein Expression. For full-length FN expression, we used the FN-YFP vector, FN-YPet/pHLSec2 in which mYPet (a YFP variant) was inserted between ${ }^{\mathrm{III}} 6$ and ${ }^{\mathrm{III}} 7$, for visualization and quantification. ${ }^{16}$ This vector has an engineered signal sequence that gives an additional three amino acids (EGS) at the $\mathrm{N}$ terminus of the secreted protein. To generate the various deletion mutants, shortened FN fragments were amplified by polymerase chain reaction and cloned back into the FN-YPet/pHLSec2 vector: $\mathrm{FN} \Delta^{\mathrm{I}} 1-5$ (deletion; $\mathrm{Q}_{32} \mathrm{AQQ} \cdots \mathrm{KCER}_{272}$ ), FN $\Delta^{\mathrm{III}} 1-3$ (deletion; $\mathrm{V}_{614}$ FIT $\cdots \mathrm{TTGT}_{901}$ ), FN $\Delta^{\mathrm{III}} 4-8$ (deletion; $\mathrm{P}_{902} \mathrm{RSD}$.. $\mathrm{RQKT}_{1447}$ ), FN $\Delta^{\mathrm{III}} 11-14$ (deletion; $\mathrm{P}_{1636} \mathrm{SQM} \cdots \mathrm{RKKT}_{2082}$ ), and FNmono (deletion; $\mathrm{C}_{2458} \mathrm{PIE} \cdots \mathrm{DSRE}_{2477}$ ). Diagrams of these mutants are shown in Figure 1. The additional restriction enzyme sites for AscI and NotI were created for cloning. For amino acid numbering, we used RefSeq NP 997647, which corresponds to that used in previous studies. ${ }^{17}$ We also created an FN-tenascin-C chimeric construct, FNtnA-D, in which FNIII domains $1-8$ in $\mathrm{FN} \Delta^{\mathrm{III}} 11-14$ were replaced with FNIII domains $\mathrm{A}-\mathrm{D}$ from tenascin- $\mathrm{C}$ (the seven main alternatively spliced FNIII domains, $\mathrm{E}_{1072}$ QAP $\cdots$ IATT $_{1708} ;$ CAA55309 was used for the numbering). A previous study showed that domains
A-D do not interact with FN. ${ }^{19}$ For co-assembly experiments, an FN-CFP construct was created by replacing mYPet in the FNYFP construct with mECFP*. ${ }^{20}$ We also generated the GFND mutants (Y973C, W1925R, and L1974R) by mutagenesis using the wild-type FN (without YFP) expression construct. ${ }^{21}$ These constructs were expressed in a transient mammalian cell expression system as reported previously. ${ }^{16,21,22}$ Briefly, purified constructs were transfected into HEK293 cells with PEI (polyethylenimine-25 kDa branched, Aldrich). The conditioned medium was collected after transfection for 6-7 days. FN, FNYFP, and the mutants were purified with a gelatin-agarose column using $4 \mathrm{M}$ urea for elution. To exchange the buffer, purified proteins were dialyzed against $\mathrm{PBS}$ \{phosphate-buffered saline [2.7 mM KCl, $1.47 \mathrm{mM} \mathrm{KH}_{2} \mathrm{PO}_{4}, 8.1 \mathrm{mM} \mathrm{Na}_{2} \mathrm{HPO}_{4}$, and $137 \mathrm{mM} \mathrm{NaCl}(\mathrm{pH} \mathrm{7.4)}]\}$. The yields of proteins varied from $\sim 0.5$ to $\sim 1.5 \mathrm{mg}$ from $\sim 100 \mathrm{~mL}$ of culture medium, obtained from three $150 \mathrm{~cm}^{2}$ tissue culture flasks. The insertion of YFP reduced protein expression levels, and some deletions or mutations decreased expression levels even further.

Glycerol Gradient Sedimentation. The sedimentation coefficients of the FN mutants were estimated by velocity sedimentation through $5 \mathrm{~mL} 15$ to $40 \%$ glycerol gradients as reported previously. ${ }^{3,7}$ Gradients contained $1 \mathrm{mM}$ PIPES ( $\mathrm{pH}$ 7.0) and either $20 \mathrm{mM} \mathrm{NaCl}$ (low salt) or $200 \mathrm{mM} \mathrm{NaCl}$ (high salt); $150 \mu \mathrm{L}$ samples were applied to the gradients and centrifuged at $38000 \mathrm{rpm}$ for $16 \mathrm{~h}$ in a Beckman SW-55 Ti rotor at $20{ }^{\circ} \mathrm{C}$. For calibration, catalase (11.3 S), aldolase (7.3 S), bovine serum albumin $(4.6 \mathrm{~S})$, and ovalbumin $(3.5 \mathrm{~S})$ were added to the samples as internal standards. Fractions were analyzed by sodium dodecyl sulfate-polyacrylamide gel electrophoresis (SDS-PAGE) (10\% acrylamide) under nonreducing conditions ( $2 \times$ loading buffer containing $10 \mathrm{mM}$ iodoacetamide). These experiments were repeated three times under both low- and high-salt conditions. The peak fractions of samples and standards were determined by the intensity of Coomassie blue- 
stained bands. For example, under low-salt conditions, FN $\Delta^{\mathrm{III}} 4-$ 8 was in fraction 4.0, catalase was in fraction 3.9, aldolase was in fraction 6.4, BSA was in fraction 9.2, and ovalbumin was in fraction 9.9 (Figure 3). On the basis of these results, a standard curve $(S=-1.2532 \times$ fraction +15.886$)$ was generated and the $S$ value of $\mathrm{FN} \Delta^{\mathrm{III}} 4-8$ was calculated to be 10.9 .

Cell Culture and FN-YFP Quantification Using YFP Fluorescence. $\mathrm{FN}(-/-)$ cells $^{23}$ were maintained with DMEM (Sigma) containing $10 \%$ fetal calf serum (FCS, Sigma). Cells were harvested with $0.05 \%$ trypsin and $2 \mathrm{mM}$ EDTA (Invitrogen) and rinsed with DMEM containing $2 \mathrm{mM}$ PMSF to quench the trypsin activity. Cells were then resuspended with DMEM containing 1\% FCS that was depleted of FN by being passed through a gelatin column. Cell suspensions $(0.4 \mathrm{~mL}, 5 \times$ $10^{5}$ cells $/ \mathrm{mL}$ ) containing $30 \mathrm{nM} \mathrm{FN-YFP} \mathrm{or} \mathrm{the} \mathrm{deletion}$ mutants were plated in a 24-well culture plate. For microscopy, $12 \mathrm{~mm}$ circular cover glasses were added to several wells, fixed, and mounted on slides after being cultured for $\sim 16 \mathrm{~h}$.

The concentration of proteins was determined from the YFP absorbance at $514 \mathrm{~nm}$ with the molar extinction coefficient for mYPet of $85000 \mathrm{M}^{-1} \mathrm{~cm}^{-1}$. ${ }^{20}$ The concentrations of FN-YFP and mutants are given for the monomer. To assay for incorporation of the YFP-labeled protein into the matrix, cells were cultured for $\sim 16 \mathrm{~h}$, and then each well was rinsed three times with $0.5 \mathrm{~mL}$ of PBS containing $\mathrm{Ca}^{2+}$ and $\mathrm{Mg}^{2+}$. Cells were then treated with 100 $\mu \mathrm{L}$ of trypsin $(10 \mu \mathrm{g} / \mathrm{mL})$ in PBS containing $5 \mathrm{mM}$ EDTA for 30 $\mathrm{min}$ at room temperature. Trypsin digestion was quenched with $2 \mathrm{mM}$ PMSF, and samples were centrifuged at $15000 \mathrm{rpm}$ for 10 min to remove the cells. The total culture YFP was then estimated from the fluorescence of the supernatant. For background subtraction, the same experiments were performed without the cells. The fluorescence was assayed with a Shimadzu RF-5301-PC spectrofluorometer. Samples were excited at 514 $\mathrm{nm}$, and emission was recorded at $528 \mathrm{~nm}$ with slit widths of 3 $\mathrm{nm}$ for excitation and $5 \mathrm{~nm}$ for emission. These measurements were performed at room temperature with quadruplicate samples in each plate. For co-assembly experiments, $15 \mathrm{nM}$ FN-CFP was mixed with $15 \mathrm{nM}$ YFP-labeled mutant FN. The concentration of FN-CFP was determined from the absorbance at $433 \mathrm{~nm}$ with a molar extinction coefficient for monomeric ECFP* of 23500 $\mathrm{M}^{-1} \mathrm{~cm}^{-1}$. After solubilization of the fluorescent proteins, the YFP fluorescence was measured as described above, while the CFP fluorescence was measured by exciting samples at $433 \mathrm{~nm}$ and recording emission at $475 \mathrm{~nm}$ with slit widths of $5 \mathrm{~nm}$ for excitation and $10 \mathrm{~nm}$ for emission. For microscopy, some samples were fixed with $3.7 \%$ formaldehyde in PBS, rinsed with PBS, and mounted on slides. Incorporation of $30 \mathrm{nM}$ recombinant $\mathrm{FN}$ or the GFND mutants was detected by immunostaining. After fixation, samples were stained with an anti-FN polyclonal antiserum (HB5, $\left.{ }^{24} 1: 1000\right)$ and an Alexa Fluor 488 anti-rabbit IgG antibody (1:1000). The samples were observed with a light microscope (Zeiss Axioplan, objective EC Plan-Neofluar $40 \times / 1.3$ oil), and the images were captured with a cooled CCD camera (CoolSNAP HQ, Roper Scientific) using AxioVision software (Zeiss) and processed with Photoshop (Adobe).

\section{RESULTS}

Conformations of the FN Deletion Mutants. The YFPtagged FN and the deletion mutants used in this study are depicted in Figure 1. The quality of the purified FN-YFP and deletion mutants was analyzed by SDS-PAGE ( $5 \%$ acrylamide) under nonreducing and reducing conditions (Figure 2). The

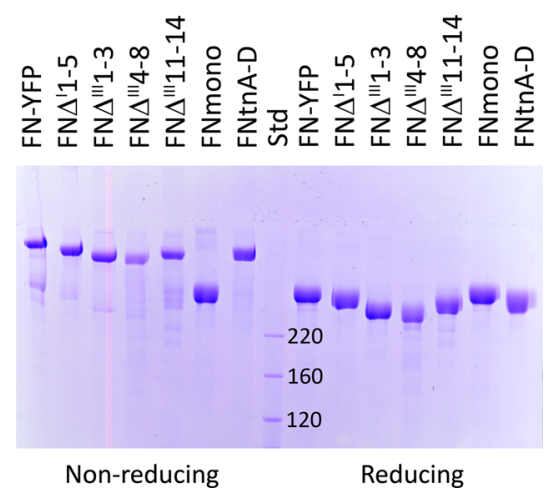

Figure 2. Quality of the FN mutants. Purified proteins were analyzed by SDS-PAGE (5\% acrylamide) under nonreducing and reducing conditions. All the proteins except FNmono formed disulfide-bonded dimers. Some minor proteolysis fragments were also seen. All mutants contained the YFP.

majority of the proteins were verified to be disulfide-bonded dimers with minor proteolysis fragments. The sedimentation coefficients for the FN mutants were determined in $1 \mathrm{mM}$ PIPES ( $\mathrm{pH} 7.0$ ) with 15 to $40 \%$ glycerol gradients containing either 20 $\mathrm{mM} \mathrm{NaCl}$ (low salt) or $200 \mathrm{mM} \mathrm{NaCl}$ (high salt) as reported previously. ${ }^{3,7}$ An earlier study by Rocco et al. ${ }^{25}$ showed that in $30 \%$ glycerol (the midpoint of our gradient) with $20 \mathrm{mM} \mathrm{NaCl}$, FN had a compact conformation similar to that at a physiological salt concentration $(150 \mathrm{mM} \mathrm{NaCl})$ with no glycerol. Similarly, in $200 \mathrm{mM} \mathrm{NaCl}$ with glycerol, $\mathrm{FN}$ has an extended conformation equivalent to $>500 \mathrm{mM} \mathrm{NaCl}$ alone. After sedimentation, the collected fractions were analyzed by SDS-PAGE (10\% acrylamide) and the sedimentation coefficients were determined by internal standards. We often saw some proteolysis fragments in this assay (the C-terminal portion of FN is particularly sensitive to protease). FN-YFP sedimented at $12.3 \mathrm{~S}$ under lowsalt conditions and at 10.0 S under high-salt conditions (Table 1 ), in agreement with previous studies showing that $\mathrm{FN}$ forms an extended conformation at a high ionic strength. ${ }^{3,7}$ These $S$ values are very similar to estimated values of 12.2 and 9.8 under lowand high-salt conditions, respectively, for recombinant FN without YFP in the study presented here (described below and in Table 2), indicating that the insertion of YFP into FN has only a minor effect on conformation.

When we tested the deletion mutants, $\mathrm{FN} \Delta^{\mathrm{III}} 1-3$ sedimented very much like FN-YFP did (Figure 3 and Table 1). Other deletion mutants, FN $\Delta^{\mathrm{I}} 1-5$ and $\mathrm{FN} \Delta^{\mathrm{III}} 4-8$ (Figure 3 ), as well as $\mathrm{FN} \Delta^{\mathrm{III}} 11-14$ and FNmono showed a small shift in sedimentation coefficient between the two salt concentrations (Table 1). FNtnA-D, in which ${ }^{\mathrm{III}} 1-8$ are replaced by seven FN domains from tenascin- $\mathrm{C}$ and which is also missing ${ }^{\mathrm{III}} 11-14$, showed a very minor shift, suggesting that the conformation of this chimeric molecule is not sensitive to salt concentration. These results indicate that domains within ${ }^{\mathrm{I}} 1-5,{ }^{\mathrm{III}} 4-8$, and ${ }^{\mathrm{III}} 11-14$ are involved in forming the compact conformation under low-salt conditions, and ${ }^{\mathrm{III}} 1-3$ appears to be expendable.

Assembly of the FN Deletion Mutants. We next examined the ability of FN deletion mutants to form a matrix, visualized by fluorescence microscopy (Figure 4A), and quantitated by measuring the YFP fluorescence solubilized by trypsin treatment (Figure $4 \mathrm{~B}$ ). FN $\Delta^{\mathrm{III}} 4-8$ and $\mathrm{FN} \Delta^{\mathrm{III}} 11-14$, in addition to $\mathrm{FN}$ YFP, showed a nice matrix fibril meshwork, although the amount of matrix was somewhat reduced, to $\sim 70 \%$ of FN-YFP. FNmono formed a very short and thin fibrillar matrix ( 10\% FN-YFP). 
Table 1. Sedimentation of the FN Deletion Mutants and the FNtnA-D Chimera

\begin{tabular}{|c|c|c|c|c|}
\hline FN mutant & $\mathrm{MW}(\mathrm{kDa})^{a}$ & $S$ in low salt, mean (three measurements) & $S$ in high salt, mean (three measurements) & change in $S(\Delta S)$ \\
\hline FN-YFP & 556 & $\begin{array}{l}\mathbf{1 2 . 3} \\
(12.4,12.2,12.4)\end{array}$ & $\begin{array}{l}\mathbf{1 0 . 0} \\
(10.0,9.9,10.0)\end{array}$ & 2.3 \\
\hline $\mathrm{FN} \Delta^{\mathrm{I}} 1-5$ & 502 & $\begin{array}{l}10.7 \\
(10.7,10.7,10.8)\end{array}$ & $\begin{array}{l}9.4 \\
(9.2,9.5,9.5)\end{array}$ & 1.3 \\
\hline $\mathrm{FN} \Delta^{\mathrm{III}} 1-3$ & 492 & $\begin{array}{l}\mathbf{1 2 . 1} \\
(12.3,12.1,12.0)\end{array}$ & $\begin{array}{l}9.6 \\
(9.7,9.5,9.7)\end{array}$ & 2.5 \\
\hline $\mathrm{FN} \Delta^{\mathrm{III}} 4-8$ & 456 & $\begin{array}{l}10.9 \\
(10.9,11.0,10.9)\end{array}$ & $\begin{array}{l}9.9 \\
(9.9,9.8,9.9)\end{array}$ & 1.0 \\
\hline $\mathrm{FN} \Delta^{\mathrm{III}} 11-14$ & 478 & $\begin{array}{l}10.4 \\
(10.2,10.4,10.5)\end{array}$ & $\begin{array}{l}9.4 \\
(9.2,9.5,9.6)\end{array}$ & 1.0 \\
\hline FNmono & 276 & $\begin{array}{l}8.5 \\
(8.2,8.5,8.8)\end{array}$ & $\begin{array}{l}7.4 \\
(7.1,7.5,7.7)\end{array}$ & 1.1 \\
\hline FNtnA-D & 455 & $\begin{array}{l}10.1 \\
(10.0,10.3,10.1)\end{array}$ & $\begin{array}{l}9.8 \\
(9.6,10.0,9.9)\end{array}$ & 0.3 \\
\hline
\end{tabular}

${ }^{a}$ Molecular weights are deduced from amino acid sequences that do not include carbohydrates and are presented for the dimer, except for FNmono.

Table 2. Sedimentation of the GFND Mutants

\begin{tabular}{|c|c|c|c|c|}
\hline & $\operatorname{MW}(\mathrm{kDa})^{a}$ & $S$ in low salt, mean (three measurements) & $S$ in high salt, mean (three measurements) & change in $S(\Delta S)$ \\
\hline FN & 501 & $\begin{array}{l}\mathbf{1 2 . 2} \\
(12.4,12.1,12.2)\end{array}$ & $\begin{array}{l}9.8 \\
(9.6,9.8,9.9)\end{array}$ & 2.4 \\
\hline $\mathrm{FN}(\mathrm{Y} 973 \mathrm{C})$ & $1002^{b}$ & $\begin{array}{l}13.0 \\
(13.0,13.2,12.8)\end{array}$ & $\begin{array}{l}11.2 \\
(11.0,11.3,11.4)\end{array}$ & 1.8 \\
\hline $\mathrm{FN}(\mathrm{W} 1925 \mathrm{R})$ & 501 & $\begin{array}{l}11.4 \\
(11.4,11.4,11.3)\end{array}$ & $\begin{array}{l}9.8 \\
(9.7,9.9,9.8)\end{array}$ & 1.6 \\
\hline $\mathrm{FN}(\mathrm{L} 1974 \mathrm{R})$ & 501 & $\begin{array}{l}\mathbf{1 1 . 3} \\
(11.3,11.4,11.3)\end{array}$ & $\begin{array}{l}9.5 \\
(9.4,9.5,9.5)\end{array}$ & 1.8 \\
\hline
\end{tabular}

${ }^{a}$ Molecular weights are deduced from amino acid sequences that do not include carbohydrates and are presented for the dimer, except for the Y973C mutant. ${ }^{b}$ The majority of the Y973C mutants were disulfide-bonded tetramers.

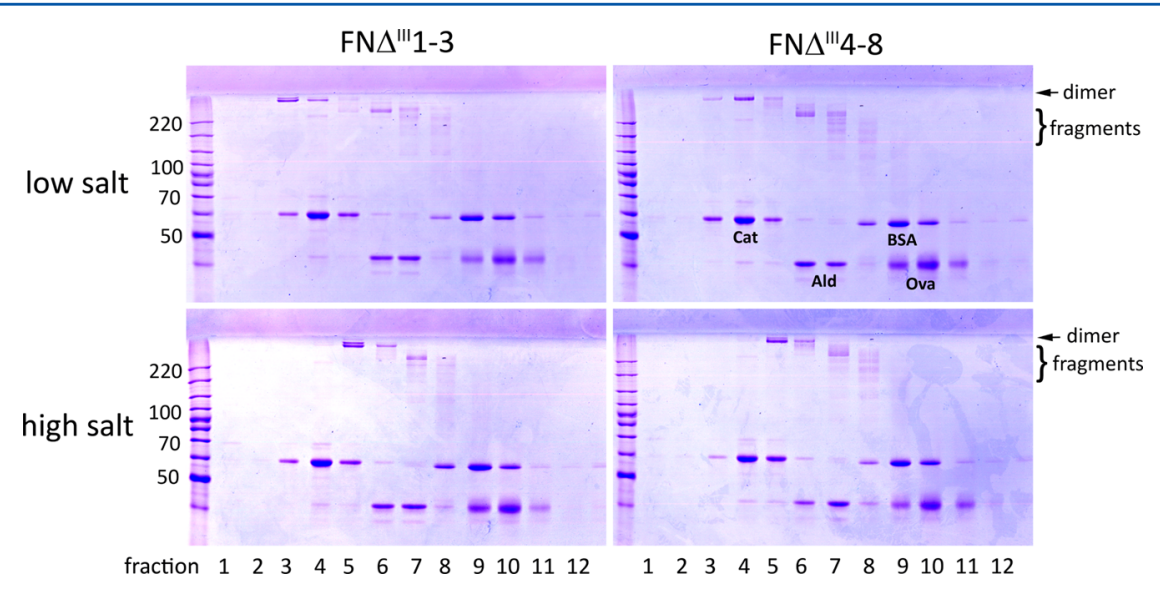

Figure 3. Glycerol gradient sedimentation of two FN deletion mutants. Fractions from the gradients were analyzed by SDS-PAGE (10\% acrylamide) under nonreducing conditions. Both mutants $\mathrm{FN} \Delta^{\mathrm{III}} 1-3$ and $\mathrm{FN} \Delta^{\mathrm{III}} 4-8$ showed a shift in sedimentation coefficient between low and high salt concentrations. Internal standards, catalase (11.3 S), aldolase (7.3 S), bovine serum albumin ( $4.6 \mathrm{~S})$, and ovalbumin (3.5 S), were used to calibrate the gradients. The dimeric FN mutants are seen in fractions 3-4 (low salt) and 5-6 (high salt). Monomeric fragments, seen in fractions 6-7 (low salt) and 7-8 (high salt), also shifted.

FN $\Delta^{\mathrm{I}} 1-5, \mathrm{FN} \Delta^{\mathrm{III}} 1-3$, and FNtnA-D formed a barely detectable matrix $(<8 \%)$, although small fibrils were occasionally seen by microscopy (Figure 4A).

Co-Assembly of the FN Deletion Mutants with FN-CFP. In the previous experiment, the deletion mutant was the only FN present. We also tested the ability of the FN mutants to coassemble with FN-CFP (as the wild type). As shown in Figure 5, FN-YFP nicely co-assembled with FN-CFP, and most of the mutants showed significant co-assembly. The assembly was quantitated by solubilizing the CFP and YFP in the matrix
(Figure 6). Most of the mutants slightly decreased the level of the FN-CFP matrix (70-95\%). In the case of FN $\Delta^{\mathrm{I}} 1-5$, however, the level of the FN-CFP matrix was increased by $\sim 15 \%$; this was the only mutant that did not co-assemble with FN-CFP. $\mathrm{FN} \Delta^{\mathrm{III}} 1-3$ and $\mathrm{FN} \Delta^{\mathrm{III}} 4-8$ co-assembled well with FN-CFP (70-80\%). In contrast, FN $\Delta^{\mathrm{III}} 11-14$, FNmono, and FNtnA-D also co-assembled, but at a reduced level ( $30-50 \%)$.

Conformations of the GFND Mutants. The quality of purified GFND mutants was analyzed by SDS-PAGE (5\% acrylamide) under nonreducing and reducing conditions (Figure 
A
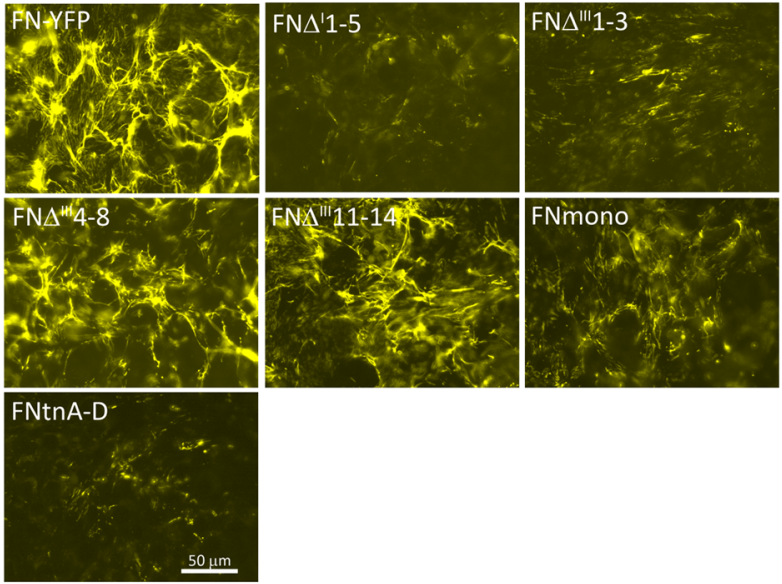

B

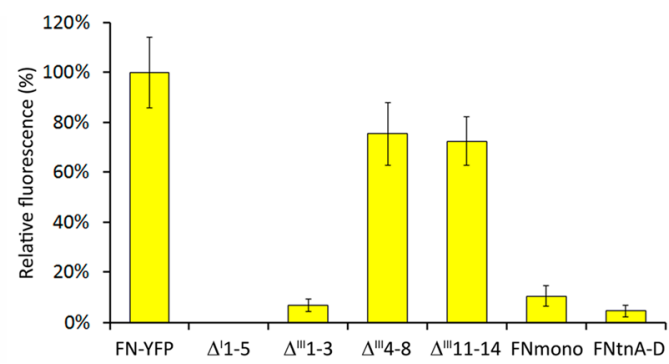

Figure 4. Fluorescence microscopy images and quantitative analysis of FN assembly by the deletion mutants. (A) FN-YFP and the deletion mutants were added to the $\mathrm{FN}(-/-)$ cell culture and incubated for $\sim 16$ h. FN $\Delta^{\mathrm{III}} 4-8$ and $\mathrm{FN} \Delta^{\mathrm{III}} 11-14$ showed fibrillar networks, although they are somewhat less than those of FN-YFP. FNmono formed very short and thin matrix fibrils. FN $\Delta^{\mathrm{I}} 1-5, \mathrm{FN} \Delta^{\mathrm{III}} 1-3$, and FNtnA-D showed almost no matrix. (B) The amount of extracellular FN was determined by measuring the YFP fluorescence after solubilizing YFP from the cell culture by trypsin treatment. The emission intensity was normalized to that of FN-YFP. All the mutants showed a decrease in the level of extracellular FN. Some mutants, such as FN $\Delta^{\mathrm{III}} 4-8$ and $\mathrm{FN} \Delta^{\mathrm{III}} 11-14$, decreased the level of $\mathrm{FN}$ to $\sim 70 \%$, while $\mathrm{FN} \Delta^{\mathrm{I}} 1-5$, FN $\Delta^{\mathrm{III}} 1-3$, FNmono, and FNtnA-D dramatically decreased the level of FN assembly. The error bars indicate the standard deviation for measurements from four wells in one experiment.

7A). The majority of the proteins were verified to be disulfidebonded dimers. However, the Y973C mutant ran primarily as a tetramer, indicating that the cysteine residue was exposed on the surface and was able to form an additional disulfide bond. The possibility of abnormal disulfide bond formation in the Y973C mutant was postulated in the original GFND study. ${ }^{17}$ When we tested the GFND mutants for matrix assembly, they all formed the matrix like wild-type FN (Figure 7B). In the case of Y973C, it formed even more matrix than wild-type FN did (Figure 7B). The sedimentation coefficients for the GFND mutants were also determined in glycerol gradients. Two of the GFND mutants, W1925R and L1974R, in which the mutations were located on III13, sedimented very much like wild-type FN under high-salt conditions (Table 2). Under low-salt conditions, however, both mutants sedimented slower than recombinant FN and FN-YFP did (Table 1), indicating that these mutations somewhat compromised the compact conformation. Interestingly, the sedimentation coefficients of the Y973C mutant, 13.0 S under low-salt conditions and 11.2 S under high-salt conditions (Figure 7C and Table 2), show that the tetramers are still capable of undergoing the compact to extended conformational change.

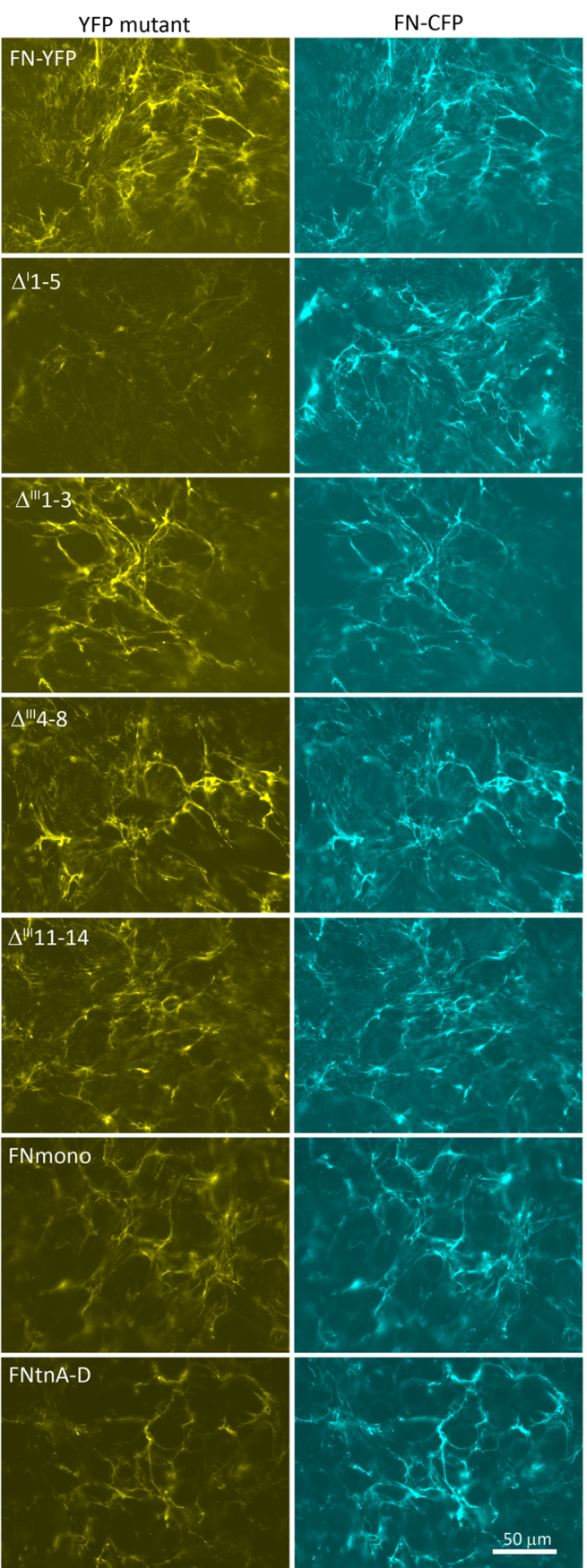

Figure 5. Fluorescence microscopy images of FN-CFP co-assembly with the deletion mutants. An equimolar mixture of FN-CFP and each YFP-labeled deletion mutant was added to the $\mathrm{FN}(-/-)$ cell culture and incubated for $\sim 16 \mathrm{~h}$. FN $\Delta^{\mathrm{I}} 1-5$ did not co-assemble with FN-CFP at all, but the other deletion mutants showed variable co-assembly.

\section{DISCUSSION}

Conformation. Johnson et al. ${ }^{7}$ proposed a model for the compact conformation of $\mathrm{FN}$, where it is formed from an interaction between ${ }^{\mathrm{III}} 2-3$ of one subunit and ${ }^{\mathrm{III}} 12-14$ of the other subunit. The work presented here confirms the importance of ${ }^{\mathrm{III}} 12-14$ for the compact conformation. However, our deletion mutant $\mathrm{FN} \Delta^{\mathrm{III}} 1-3$, which lacked domains ${ }^{\mathrm{III}} 2-3$, formed the compact conformation as well as full-length $\mathrm{FN}$, suggesting that ${ }^{\mathrm{III}} 2-3$ is not an essential interaction. Recently, it 

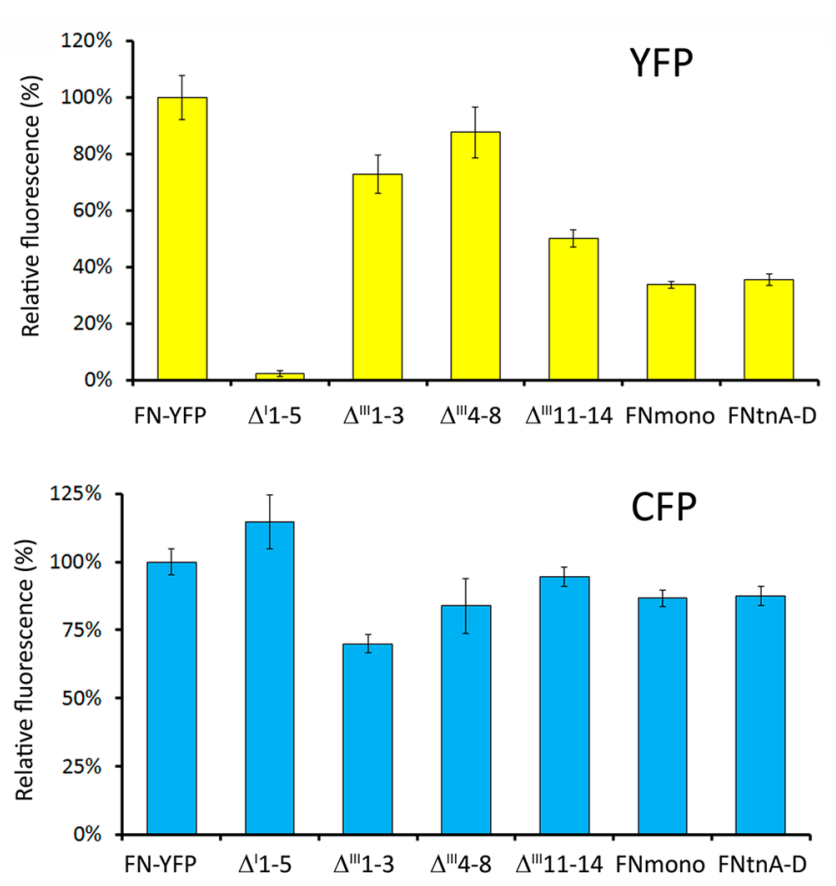

Figure 6. Quantitative analysis of FN-CFP co-assembly with the deletion mutants. The amount of extracellular FN was determined by measuring the CFP and YFP fluorescence after solubilizing FPs from cell culture by trypsin treatment. CFP and YFP values were normalized to each of the emission intensities from the mixture of FN-CFP and FNYFP. The error bars indicate the standard deviation for measurements from four wells in one experiment.

has been reported that the binding of a bacterial adhesin to ${ }^{\mathrm{I}} 1-5$ induces an extended conformation. ${ }^{26}$ This study confirmed that I $1-5$ contributes to the compact conformation, because deleting it reduced the magnitude of the change. Deletion of ${ }^{\mathrm{III}} 4-8$ or ${ }^{\mathrm{III}} 11-14$ had stronger effects, but all of these deletion mutants still showed a conformational change. This suggests that there are multiple interactions involved in forming the compact conformation. The existence of multiple FN conformations has been proposed previously on the basis of a monoclonal antibody that was sensitive to conformation. ${ }^{6,10}$

In our sedimentation analysis, FNmono showed a change in sedimentation from low- to high-salt conditions (Table 1). Sedimentation shifts were also seen in the proteolytic fragments of $\mathrm{FN} \Delta^{\mathrm{III}} 1-3$ and $\mathrm{FN} \Delta^{\mathrm{III}} 4-8$, which were presumably monomeric (Figure 3). This could be due to a conformational change within the monomer as suggested by Erickson and Carrell $^{3}$ or it could be due to dimerization of the monomer under low-salt conditions as reported by Johnson et al. ${ }^{7}$ For the FN dimer, however, the sedimentation shift is probably due to binding of one subunit to its partner, accommodated by bending at hinge points and resulting in the compact conformation. On the basis of the sedimentation coefficients of the FN monomer and FN dimer under low-salt conditions, a dimeric form produced by association of two FN monomers is unlikely to be as compact as the disulfide-bonded FN dimer.

Assembly. The laboratories of Schwarzbauer ${ }^{27-29}$ and Sekiguchi ${ }^{30-32}$ pioneered the study of FN assembly by using FN deletion mutants. Most of our results are consistent with their earlier findings. Both agree that ${ }^{\mathrm{I}} 1-5$ is crucial for assembly, and we now show additionally that FN lacking ${ }^{\mathrm{I}} 1-5$ cannot even co-assemble with full-length FN. In addition to ${ }^{\mathrm{I}} 1-5,{ }^{\mathrm{III}} 1-3$ also appears to be important for matrix assembly, because the mutants lacking these domains (FN $\Delta^{\mathrm{III}} 1-3$ and $\left.\mathrm{FNtn} A-\mathrm{D}\right)$ did not form a matrix. This is consistent with the earlier study of Sechler et al. ${ }^{29}$ who showed that deletion of ${ }^{\mathrm{III}} 1-2$ or ${ }^{\mathrm{III}} 2-5$ substantially compromised matrix assembly. They also showed that deletion of ${ }^{\mathrm{III}} 1$ did not affect assembly, strongly implicating $\mathrm{III}_{2}$ as the important domain.

Domain ${ }^{\mathrm{III}} 2$ is now a prime candidate for an essential role in matrix assembly, perhaps by binding the other essential matrix assembly site, ${ }^{\mathrm{I}} 1-5$. We showed previously that ${ }^{\mathrm{II}} 2$ could bind ${ }^{\mathrm{I}} 1-5$ in solution, but only when ${ }^{\mathrm{III}} 2$ was destabilized by removing the G strand. Sechler et al. ${ }^{29}$ showed that ${ }^{\mathrm{III}} 2$ coated on plastic could bind whole $\mathrm{FN}$ or the $70 \mathrm{kDa}$ fragment $\left({ }^{\mathrm{I}} 1-5-{ }^{\mathrm{II}} 1-2-{ }^{\mathrm{I}} 6-\right.$ 9 ). The adsorption to plastic may have partially denatured ${ }^{\mathrm{III}} 2$, exposing the binding site for ${ }^{\mathrm{I}} 1-5$. We also found previously that introducing a disulfide bond in ${ }^{\mathrm{II}} 2$, which stabilized folding, significantly reduced the level of the FN matrix. ${ }^{16}$ The binding of the $\mathrm{N}$-terminal region, ${ }^{\mathrm{I}} 1-5$, to ${ }^{\mathrm{III}} 2$ also agrees with the $\mathrm{N}$ terminal $30-40 \mathrm{~nm}$ overlap between FN molecules in matrix fibrils observed by super-resolution microscopy. ${ }^{33}$

All constructs that contained a ${ }^{\mathrm{I}} 1-5$ segment could coassemble with wild-type FN. This suggests that the primary event in matrix assembly is binding of ${ }^{\mathrm{I}} 1-5$ on the soluble $\mathrm{FN}$ to an FNIII domain in the already assembled matrix, but this binding event apparently cannot go in the opposite direction. For instance, ${ }^{\mathrm{I}} 1-5$ from wild-type FN already incorporated in the matrix cannot bind FNIII domains on soluble FN $\Delta^{\mathrm{I}} 1-5$. This is consistent with the idea of cryptic binding sites that are exposed only in matrix fibrils under tension. ${ }^{34,35}$ Specifically, the FNIII sites that bind ${ }^{\mathrm{I}} 1-5$ are inactive in soluble FN dimers and are activated by a conformational change and/or domain unfolding, induced by tension or another mechanism, when $\mathrm{FN}$ is incorporated into matrix fibrils.

Fibronectin Glomerulopathy (GFND). Castelletti et al. ${ }^{17}$ generated recombinant heparin binding fragments containing III $12-14$ with GFND mutations (W1925R or L1974R) in ${ }^{\mathrm{III}} 13$ and found that these mutations significantly reduced the level of heparin binding. Both mutations substitute buried hydrophobic core residues with arginine. Therefore, these mutants are likely to have folding problems. The other mutation, Y973C in ${ }^{\mathrm{III}} 4$, was not characterized in the original report. The tyrosine residue is at the beginning of the F strand and is highly conserved in FNIII domains. It helps stabilize the domain by forming a tyrosine corner, ${ }^{36}$ and the corresponding tyrosine residues in crystallized FNIII domains ${ }^{37,38}$ are mostly buried. Our study shows that the Y973C mutant forms additional disulfide bonds, indicating that the residue is exposed on the surface by misfolding. When we tested the conformations of the full-length GFND mutants, the W1925R and L1974R mutants formed slightly less of the compact conformation under low-salt conditions. The mutations did not affect matrix assembly dramatically. Unfortunately, these results do not provide any direct explanation for how these mutations cause GFND.

The originally reported GFND mutations from patients were heterozygous missense mutations, while our recombinant GFND mutants are homogeneous dimers. Therefore, the GFND mutations in patients should have milder effects on conformation. In addition, there is no evidence indicating that the GFND mutants cause thrombosis by misfolding of FN in patients. We previously reported that the folding and stability of FNIII domains were crucial for anastellin-induced FN aggregation. ${ }^{15,16}$ We suggest that the GFND mutations destabilize the FNIII domains and these unstable FNIII domains 

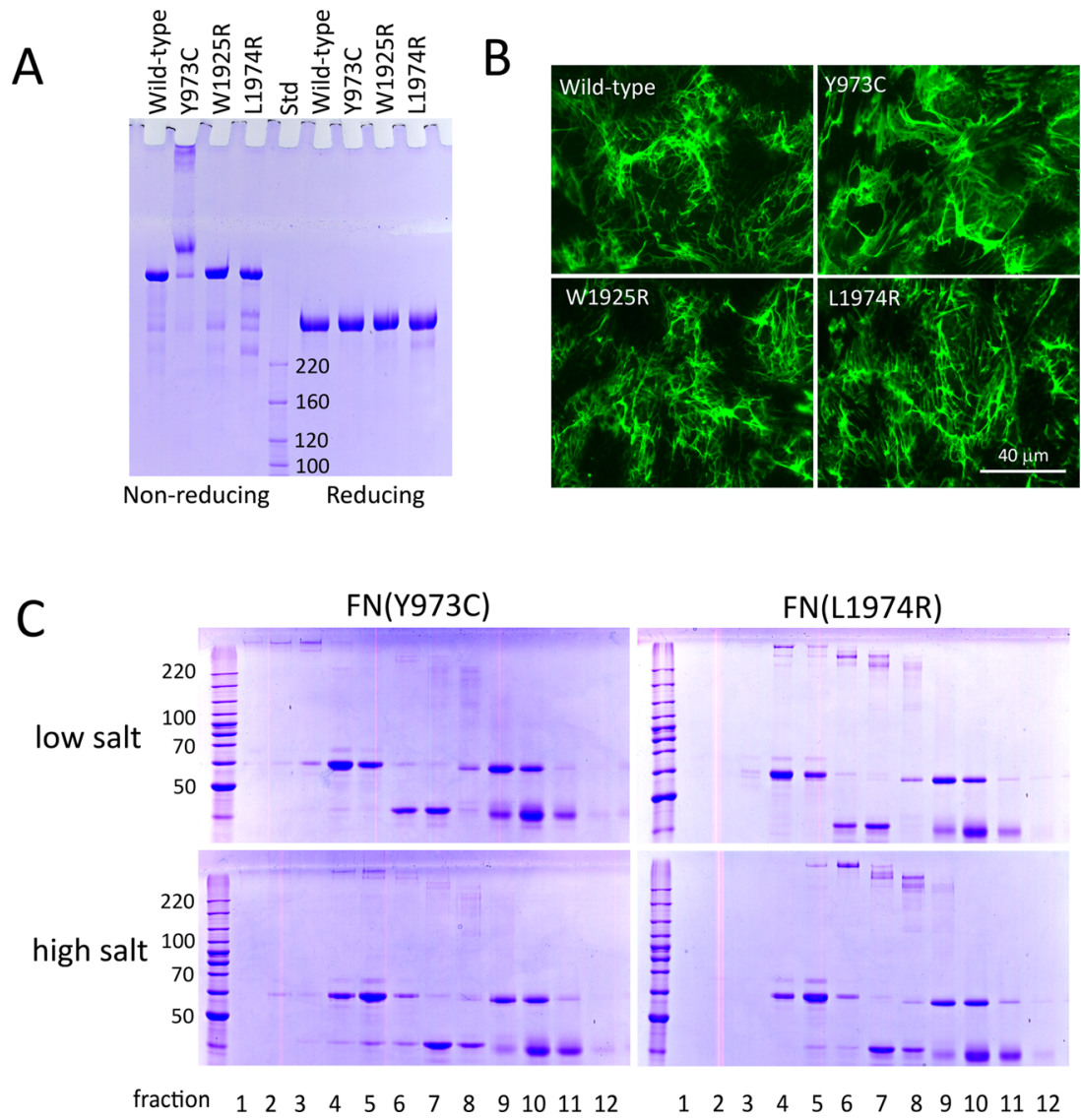

Figure 7. Analysis of the GFND mutants. (A) Purified proteins were analyzed by SDS-PAGE (5\% acrylamide) under nonreducing and reducing conditions. The W1925R and L1974R mutants migrated as dimers like wild-type FN; the majority of the Y973C mutant migrated as tetramers, and some higher multimers were also seen on the stacking gel. Under reducing conditions, the Y973C mutant migrated as monomers, indicating that the tetramers and higher multimers were formed by disulfide bonds. (B) The GFND mutants were added to the FN $(-/-)$ cell culture and incubated for $\sim 16 \mathrm{~h}$. (C) Fractions from the glycerol gradients were analyzed by SDS-PAGE (10\% acrylamide) under nonreducing conditions. Both mutants Y973C and L1974R showed a shift in sedimentation coefficient between low- and high-salt conditions. The L1974R mutant showed additional small fragments, indicating that it is sensitive to protease. Internal standards, catalase (11.3 S), aldolase (7.3 S), bovine serum albumin (4.6 S), and ovalbumin (3.5 S), were used to calibrate the gradients.

may slowly form nonfibrillar aggregates in the kidney. In the case of the Y973C mutant, it is also possible that unusual disulfide bond formation, even in the case of a heterozygous mutation, enhances nonfibrillar aggregation.

\section{AUTHOR INFORMATION}

\section{Corresponding Authors}

*T.O.: Department of Cell Biology, Box 3709, Duke University Medical Center, Durham, NC 27710. Telephone: 919-684-6385.

Fax: 919-684-8090. E-mail: tomoo.ohashi@duke.edu.

*H.P.E.: E-mail: harold.erickson@duke.edu.

\section{ORCID $\odot$}

Tomoo Ohashi: 0000-0001-8254-0526

\section{Present Address}

${ }^{\dagger}$ C.A.L.: Department of Biomedical Engineering, Virginia Commonwealth University, Box 843607, Richmond, VA 23284-3067.

\section{Funding}

This work was supported in whole or part by National Institutes of Health Grant CA047056.

\section{Notes}

The authors declare no competing financial interest.

\section{ABBREVIATIONS}

FN, fibronectin; GFND, glomerulopathy with fibronectin deposits; FNIII, fibronectin type III; PBS, phosphate-buffered saline; PMSF, phenylmethanesulfonyl fluoride; YFP, yellow fluorescent protein; CFP, cyan fluorescent protein; CCD, charge-coupled device.

\section{REFERENCES}

(1) Tamkun, J. W., and Hynes, R. O. (1983) Plasma fibronectin is synthesized and secreted by hepatocytes. J. Biol. Chem. 258, 4641-4647.

(2) Schwarzbauer, J. E., Spencer, C. S., and Wilson, C. L. (1989) Selective secretion of alternatively spliced fibronectin variants. J. Cell Biol. 109, 3445-3453.

(3) Erickson, H. P., and Carrell, N. A. (1983) Fibronectin in extended and compact conformations. Electron microscopy and sedimentation analysis. J. Biol. Chem. 258, 14539-14544.

(4) Lai, C. S., Tooney, N. M., and Ankel, E. G. (1984) Structure and flexibility of plasma fibronectin in solution: electron spin resonance spin-label, circular dichroism, and sedimentation studies. Biochemistry 23, 6393-6397.

(5) Williams, E. C., Janmey, P. A., Ferry, J. D., and Mosher, D. F. (1982) Conformational states of fibronectin. Effects of $\mathrm{pH}$, ionic strength, and collagen binding. J. Biol. Chem. 257, 14973-14978.

(6) Ugarova, T. P., Zamarron, C., Veklich, Y., Bowditch, R. D., Ginsberg, M. H., Weisel, J. W., and Plow, E. F. (1995) Conformational 
transitions in the cell binding domain of fibronectin. Biochemistry 34, 4457-4466.

(7) Johnson, K. J., Sage, H., Briscoe, G., and Erickson, H. P. (1999) The compact conformation of fibronectin is determined by intramolecular ionic interactions. J. Biol. Chem. 274, 15473-15479.

(8) Homandberg, G. A., and Erickson, J. W. (1986) Model of fibronectin tertiary structure based on studies of interactions between fragments. Biochemistry 25, 6917-6925.

(9) Vakonakis, I., Staunton, D., Ellis, I. R., Sarkies, P., Flanagan, A., Schor, A. M., Schor, S. L., and Campbell, I. D. (2009) Motogenic sites in human fibronectin are masked by long range interactions. J. Biol. Chem. 284, 15668-15675.

(10) Maurer, L. M., Ma, W., Eickstaedt, N. L., Johnson, I. A., TomasiniJohansson, B. R, Annis, D. S., and Mosher, D. F. (2012) Ligation of the fibrin-binding domain by beta-strand addition is sufficient for expansion of soluble fibronectin. J. Biol. Chem. 287, 13303-13312.

(11) Sakai, T., Johnson, K. J., Murozono, M., Sakai, K., Magnuson, M. A., Wieloch, T., Cronberg, T., Isshiki, A., Erickson, H. P., and Fassler, R. (2001) Plasma fibronectin supports neuronal survival and reduces brain injury following transient focal cerebral ischemia but is not essential for skin-wound healing and hemostasis. Nat. Med. 7, 324-330.

(12) Ni, H., Yuen, P. S., Papalia, J. M., Trevithick, J. E., Sakai, T., Fassler, R., Hynes, R. O., and Wagner, D. D. (2003) Plasma fibronectin promotes thrombus growth and stability in injured arterioles. Proc. Natl. Acad. Sci. U. S. A. 100, 2415-2419.

(13) Chen, H., and Mosher, D. F. (1996) Formation of sodium dodecyl sulfate-stable fibronectin multimers. Failure to detect products of thioldisulfide exchange in cyanogen bromide or limited acid digests of stabilized matrix fibronectin. J. Biol. Chem. 271, 9084-9089.

(14) Ohashi, T., and Erickson, H. P. (2009) Revisiting the mystery of fibronectin multimers: the fibronectin matrix is composed of fibronectin dimers cross-linked by non-covalent bonds. Matrix Biol. 28, 170-175.

(15) Ohashi, T., and Erickson, H. P. (2005) Domain unfolding plays a role in superfibronectin formation. J. Biol. Chem. 280, 39143-39151.

(16) Ohashi, T., and Erickson, H. P. (2011) Fibronectin aggregation and assembly: unfolding of the second fibronectin type III domain. J. Biol. Chem. 286, 39188-39199.

(17) Castelletti, F., Donadelli, R., Banterla, F., Hildebrandt, F., Zipfel, P. F., Bresin, E., Otto, E., Skerka, C., Renieri, A., Todeschini, M., Caprioli, J., Caruso, R. M., Artuso, R., Remuzzi, G., and Noris, M. (2008) Mutations in FN1 cause glomerulopathy with fibronectin deposits. Proc. Natl. Acad. Sci. U. S. A. 105, 2538-2543.

(18) Ohtsubo, H., Okada, T., Nozu, K., Takaoka, Y., Shono, A., Asanuma, K., Zhang, L., Nakanishi, K., Taniguchi-Ikeda, M., Kaito, H., Iijima, K., and Nakamura, S. I. (2016) Identification of mutations in FN1 leading to glomerulopathy with fibronectin deposits. Pediatr. Nephrol. 31, 1459-1467.

(19) Chung, C. Y., Zardi, L., and Erickson, H. P. (1995) Binding of tenascin-C to soluble fibronectin and matrix fibrils. J. Biol. Chem. 270, 29012-29017.

(20) Ohashi, T., Galiacy, S. D., Briscoe, G., and Erickson, H. P. (2007) An experimental study of GFP-based FRET, with application to intrinsically unstructured proteins. Protein Sci. 16, 1429-1438.

(21) Lemmon, C. A., Ohashi, T., and Erickson, H. P. (2011) Probing the folded state of fibronectin type III domains in stretched fibrils by measuring buried cysteine accessibility. J. Biol. Chem. 286, 2637526382.

(22) Aricescu, A. R., Lu, W., and Jones, E. Y. (2006) A time- and costefficient system for high-level protein production in mammalian cells. Acta Crystallogr., Sect. D: Biol. Crystallogr. 62, 1243-1250.

(23) Saoncella, S., Echtermeyer, F., Denhez, F., Nowlen, J. K., Mosher, D. F., Robinson, S. D., Hynes, R. O., and Goetinck, P. F. (1999) Syndecan-4 signals cooperatively with integrins in a Rho-dependent manner in the assembly of focal adhesions and actin stress fibers. Proc. Natl. Acad. Sci. U. S. A. 96, 2805-2810.

(24) Ohashi, T., Kiehart, D. P., and Erickson, H. P. (2002) Dual labeling of the fibronectin matrix and actin cytoskeleton with green fluorescent protein variants. J. Cell Sci. 115, 1221-1229.
(25) Rocco, M., Carson, M., Hantgan, R., McDonagh, J., and Hermans, J. (1983) Dependence of the shape of the plasma fibronectin molecule on solvent composition. Ionic strength and glycerol content. J. Biol. Chem. 258, 14545-14549.

(26) Harris, G., Ma, W., Maurer, L. M., Potts, J. R., and Mosher, D. F. (2014) Borrelia burgdorferi protein BBK32 binds to soluble fibronectin via the $\mathrm{N}$-terminal $70-\mathrm{kDa}$ region, causing fibronectin to undergo conformational extension. J. Biol. Chem. 289, 22490-22499.

(27) Schwarzbauer, J. E. (1991) Identification of the fibronectin sequences required for assembly of a fibrillar matrix. J. Cell Biol. 113, $1463-1473$.

(28) Sechler, J. L., Takada, Y., and Schwarzbauer, J. E. (1996) Altered rate of fibronectin matrix assembly by deletion of the first type III repeats. J. Cell Biol. 134, 573-583.

(29) Sechler, J. L., Rao, H., Cumiskey, A. M., Vega-Colon, I., Smith, M. S., Murata, T., and Schwarzbauer, J. E. (2001) A novel fibronectin binding site required for fibronectin fibril growth during matrix assembly. J. Cell Biol. 154, 1081-1088.

(30) Ichihara-Tanaka, K., Maeda, T., Titani, K., and Sekiguchi, K. (1992) Matrix assembly of recombinant fibronectin polypeptide consisting of amino-terminal $70 \mathrm{kDa}$ and carboxyl-terminal $37 \mathrm{kDa}$ regions. FEBS Lett. 299, 155-158.

(31) Ichihara-Tanaka, K., Titani, K., and Sekiguchi, K. (1995) Role of the carboxyl-terminal Fib2 domain in fibronectin matrix assembly. J. Cell Sci. 108 (Part 3), 907-915.

(32) Kozaki, T., Matsui, Y., Gu, J., Nishiuchi, R., Sugiura, N., Kimata, K., Ozono, K., Yoshikawa, H., and Sekiguchi, K. (2003) Recombinant expression and characterization of a novel fibronectin isoform expressed in cartilaginous tissues. J. Biol. Chem. 278, 50546-50553.

(33) Fruh, S. M., Schoen, I., Ries, J., and Vogel, V. (2015) Molecular architecture of native fibronectin fibrils. Nat. Commun. 6, 7275.

(34) Halliday, N. L., and Tomasek, J. J. (1995) Mechanical properties of the extracellular matrix influence fibronectin fibril assembly in vitro. Exp. Cell Res. 217, 109-117.

(35) Zhong, C., Chrzanowska-Wodnicka, M., Brown, J., Shaub, A., Belkin, A. M., and Burridge, K. (1998) Rho-mediated contractility exposes a cryptic site in fibronectin and induces fibronectin matrix assembly. J. Cell Biol. 141, 539-551.

(36) Hamill, S. J., Cota, E., Chothia, C., and Clarke, J. (2000) Conservation of folding and stability within a protein family: the tyrosine corner as an evolutionary cul-de-sac. J. Mol. Biol. 295, 641-649.

(37) Leahy, D. J., Aukhil, I., and Erickson, H. P. (1996) 2.0 A crystal structure of a four-domain segment of human fibronectin encompassing the RGD loop and synergy region. Cell 84, 155-164.

(38) Sharma, A., Askari, J. A., Humphries, M. J., Jones, E. Y., and Stuart, D. I. (1999) Crystal structure of a heparin- and integrin-binding segment of human fibronectin. ЕМBO J. 18, 1468-1479. 